\title{
Localization and characterization of the hypothetical protein CT440 in Chlamydia trachomatis-infected cells
}

\author{
LI Zhong $\mathrm{Yu}^{1 \dagger}$, HUANG QiuLin ${ }^{2 \dagger}$, SU ShengMei ${ }^{1}$, ZHOU Zhou $^{1}$, CHEN ChaoQun ${ }^{1}$, \\ ZHONG GuangMing ${ }^{3} \&$ WU YiMou ${ }^{*}$ \\ ${ }^{1}$ Department of Microbiology and Immunology, School of Medicine, University of South China, Hengyang 421001, China; \\ ${ }^{2}$ Department of General Surgery, the First Affiliated Hospital of University of South China, Hengyang 421001, China; \\ ${ }^{3}$ Department of Microbiology and Immunology, University of Texas Health Science Center, San Antonio, TX 78229, USA
}

Received October 4, 2011; accepted November 15, 2011

\begin{abstract}
The inclusion membrane proteins play potentially important roles in chlamydial biology and pathogenesis. Here we localized and characterized the hypothetical protein CT440 in Chlamydia trachomatis-infected cells. The open reading frame (ORF) encoding the CT440 protein from the $C$. trachomatis serovar D genome was cloned into the prokaryotic expression vector pGEX-6p and expressed as a glutathione-S-transferase (GST) fusion protein in E. coli XL1-Blue. The CT440 fusion protein was used to immunize mice to raise antigen-specific antibody. After verification by Western blot and immunofluorescence assay (IFA), the specific antibody was used to localize the endogenous CT440 protein and to detect its expression pattern in Chlamydia-infected cells. Cytosolic expression of CT440 in HeLa cells was also carried out to evaluate the effect of the CT440 protein on the subsequent chlamydial infection. The results showed that the hypothetical protein CT440 was localized in the $C$. trachomatis inclusion membrane, and was detectable $12 \mathrm{~h}$ after chlamydial infection. Expression of CT440 in the cytoplasm did not inhibit the subsequent chlamydial infection. In summary, we have identified a new inclusion membrane protein that may be an important candidate for understanding C. trachomatis pathogenesis.
\end{abstract}

Chlamydia trachomatis, CT440, inclusion membrane protein

Citation: $\quad$ Li Z Y, Huang Q L, Su S M, et al. Localization and characterization of the hypothetical protein CT440 in Chlamydia trachomatis-infected cells. Sci China Life Sci, 2011, 54: 1048-1054, doi: 10.1007/s11427-011-4243-1

Chlamydia trachomatis is a gram-negative obligate intracellular bacterial pathogen that infects a wide range of cell types including those of the eye and genital tract epithelia. Ocular infection is the leading cause of preventable blindness worldwide [1], and urogenital tract infection remains the most prevalent cause of sexually transmitted diseases in the developed countries resulting in pelvic inflammatory disease, ectopic pregnancy, and infertility [2,3]. Chlamydial genital tract infection may also significantly increase the risk for HIV infection and cervical squamous cell carcinoma

${ }^{\dagger}$ Contributed equally to this work

*Corresponding author (email: wym1004@sina.com)
[4-7]. The majority of chlamydial infections are either asymptomatic or have mild symptoms; infected individuals can also serve as important reservoirs for new infections.

C. trachomatis has a unique biphasic developmental cycle within a membrane-bound vacuole termed an inclusion $[8,9]$. The cycle starts with the attachment of the infectious, metabolically inactive elementary body (EB) to a eukaryotic host cell. The infectious EBs differentiate immediately to metabolically active bacteria forming noninfectious reticulate bodies (RBs). At the end of the developmental cycle, the progeny RBs differentiate back into EBs that are released to the extracellular space to initiate a new infectious cycle [10]. To establish and maintain a successful in- 
travacuolar growth, $C$. trachomatis has to acquire nutrients and metabolic intermediates from host cells $[11,12]$ and has to secrete chlamydial products into host cell cytoplasm [13-16]. The chlamydial proteins localized in the inclusion membrane (designated as Inc proteins) are hypothesized to play important roles in chlamydial interactions with host cells. Based on computationally generated hydropathy profiles, the CT440 protein was predicted to localize in the inclusion membrane, but not all chlamydial proteins are predicted. Therefore, identification and characterization of the predicted inclusion membrane proteins may provide important information for understanding chlamydial pathogenic mechanisms.

In the present study, using an anti-fusion protein antibody approach, we have systematically evaluated the localization of the putative inclusion membrane protein CT440 in C. trachomatis-infected cells. We investigated the distribution pattern of CT440 and its effect on subsequent chlamydial infection. We found that the hypothetical protein CT440 was localized in the inclusion membrane of Chlamydia-infected cells and was detectable $12 \mathrm{~h}$ after chlamydial infection. Further characterization of the CT440 protein revealed that the pre-existing cytosolic CT440 fusion protein did not affect the subsequent Chlamydia infection. These observations suggested that the CT440 protein may represent an important candidate for understanding $C$. trachomatis pathogenesis.

\section{Materials and methods}

\subsection{Cell culture and chlamydial infection}

HeLa cells (ATCC catalog No. CCL2) were grown on glass coverslips overnight prior to chlamydial inoculation. $C$. trachomatis serovar D diluted in Dulbecco's modified Eagle's medium (DMEM) (Gibco BRL, Rockville, MD) with $10 \%$ heat-inactivated fetal calf serum (FCS) (Gibco BRL) was directly inoculated onto the cell monolayers. The cell samples were cultured at $37^{\circ} \mathrm{C}$ in a $\mathrm{CO}_{2}$ incubator and processed at various time points after infection for immunoassays.

\subsection{CT440 gene cloning and fusion protein expression}

The ORF that codes for the hypothetical protein CT440 from the $C$. trachomatis serovar D genome was amplified with 5'-CGCGGATCCATGAAAGTTGTTGTGAATCCTAC-3' and 5'-TTTTCCTTTTGCGGCCGCTTATTTTTCTTTTGTGACAAGAAAAT-3' as the forward and reverse primers respectively. The underlined letters indicate BamH I and Not I (Sigma, St. Louis, MO) restriction enzyme sites. The CT440 gene was cloned into the pGEX-6p vector (Amersham Pharmacia Biotech, Inc., Piscataway, NJ) to construct the recombinant plasmid pGEX-6p/CT440. The recombinant plasmid was transformed into $E$. coli XL1-Blue host cells to express the fusion protein with glutathioneS-transferase (GST) fused to the $\mathrm{N}$ terminus of the CT440 protein. Expression of the fusion protein was induced with isopropyl- $\beta$-D-thiogalactopyranoside (IPTG) (Invitrogen, Carlsbad, CA) and the fusion protein was extracted by lysing the bacteria via sonication in a Triton-X100 lysis buffer (1\% Triton X-100, 1 mmol L ${ }^{-1}$ phenylmethylsulfonyl fluoride, 75 units $\mathrm{mL}^{-1}$ of aprotinin, $20 \mu \mathrm{mol} \mathrm{L} \mathrm{L}^{-1}$ leupeptin, and $1.6 \mu \mathrm{mol} \mathrm{L}{ }^{-1}$ pepstatin). After high-speed centrifugation to remove debris, the fusion protein was further purified using glutathione-conjugated agarose beads (Pharmacia) for the subsequent experiments.

\subsection{Mice immunization and antibody production}

For antibody production, the purified fusion protein was used to immunize mice as described previously [17]. Briefly, female BALB/c mice (4-6 weeks old) were first immunized intraperitoneally with $100 \mu \mathrm{g}$ of purified fusion protein emulsified with complete Freund's adjuvant (Sigma). Subsequently, the mice were given two injections with $50 \mu \mathrm{g}$ of purified fusion protein emulsified with incomplete Freund's adjuvant (Sigma) at 2-week intervals. One week after the final injection, mice sera were screened for polyclonal antibody to CT440 protein and after the titer of the specific antibody reached 1:2000 or higher, the mice were sacrificed. Mice sera were collected and stored in 50\% glycerol at $-20^{\circ} \mathrm{C}$.

\subsection{SDS-PAGE and Western blotting}

The purified fusion protein GST-CT440 was solubilized in $2 \times$ SDS sample buffer and loaded on $12 \%$ SDS-polyacrylamide gel. As controls the same amount of GST-IncA (an inclusion membrane protein), GST-CPAF (chlamydial protease-like activity factor, a secreted protein), and GST-HSP60 (an intra-inclusion protein) were loaded on the same gel. After electrophoresis, the proteins were transferred to a nitrocellulose membrane. The membrane was blocked with 5\% non-fat milk and incubated with anti-CT440 fusion protein antibody as primary antibody for $2 \mathrm{~h}$ at room temperature. The membrane was then washed with phosphate buffered saline (PBS) containing 0.05\% Tween 20 (Sigma) and incubated with goat anti-mouse horseradish peroxidase-conjugated secondary antibody (Jackson ImmunoResearch Laboratories Inc., West Grove, PA) for $1 \mathrm{~h}$. Finally, the proteins were detected using an enhanced chemiluminescence (ECL) kit (Santa Cruz Biotechnology, Inc., Santa Cruz, CA).

\subsection{Immunofluorescence assay}

Immunofluorescence assay (IFA) was performed as previously described $[14,18]$. HeLa cells infected with $C$. trachomatis serovar D were fixed with $4 \%$ paraformaldehyde 
(Sigma) in PBS for $15 \mathrm{~min}$ at room temperature, followed by permeabilization with $0.1 \%$ Triton $\mathrm{X}-100$ for an additional $4 \mathrm{~min}$. After being washed and blocked, the cell samples were subjected to various combinations of antibodies and chemical staining. A rabbit anti-chlamydial antibody and a goat anti-rabbit immunoglobulin $\mathrm{G}(\mathrm{IgG})$ secondary antibody conjugated with Cy2 (Jackson ImmunoResearch) were used to visualize chlamydial inclusions; mouse antibodies against the CT440 protein, IncA, HSP60, CPAF and goat anti-mouse IgG conjugated with Cy3 (Jackson ImmunoResearch) were used to visualize the corresponding antigens; Hoechst 33258 (Sigma) was used to visualize nuclear DNA. In some cases, the anti-fusion protein antibody was preabsorbed with either the GST-CT440 or GST-IncA fusion protein immobilized onto agarose beads prior to the staining of the cell samples. The cell samples with immuno-labeling were subjected to image analysis and acquisition using an AX-70 fluorescence microscope (Olympus, Melville, NY, USA) as described elsewhere [19,20].

\subsection{Eukaryotic expression of the CT440 protein in mammalian cells}

The ORF that codes for the CT440 protein was cloned into the pDSRed-Monomer-C1 mammalian expression vector (BD Biosciences Clontech, San Jose, CA) and expressed as a fusion protein with red fluorescence protein (RFP) fused to the N-terminus of CT440. The recombinant plasmid was transfected into HeLa cells using Lipofectamine 2000 transfection reagent according to the protocol of the manufacturer (Invitrogen). The RFP fusion protein was visualized via either the fusion tag RFP or the mouse anti-CT440 protein antibody. In some experiments, the transfected cells were infected with $C$. trachomatis serovar D $12 \mathrm{~h}$ posttransfection, and processed for visualization of the transfection and infection via an IFA as described above, to assess the effects of the RFP-CT440 fusion protein on the subsequent chlamydial infection.

\subsection{Statistical analysis}

Data from three independent experiments are presented as the mean \pm standard error. The statistical significance of the differences between groups was assessed by the Student's $t$-test. $P$-values $<0.05$ were considered as statistically significant.

\section{Results}

\subsection{Expression and purification of the CT440 fusion protein}

The gene encoding CT440 was cloned into the prokaryotic expression vector pGEX-6p and the recombinant plasmid
pGEX-6p/CT440 was transformed into an expression host $E$. coli XL1-Blue. After being induced with IPTG and purified with glutathione agarose beads, the fusion protein (molecular weight of $38.6 \mathrm{kD}$ ) was observed on SDS-PAGE; however, this band was absent in E. coli XL1-Blue cells that had been transformed with the blank vector (Figure 1A), suggesting that CT440 was successfully expressed as the GST fusion protein. This result was further confirmed by Western blot assay using anti-GST MAb (Figure 1B).

\subsection{Specificity of the antibody raised with the GST-CT440 fusion protein}

To determine the location of the hypothetical protein CT440 during chlamydial infection, we used the CT440 fusion protein to immunize mice to generate antibodies against CT440. When it was properly diluted, the anti-fusion protein antibody only recognized the CT440 fusion protein and had no obvious cross-reactivity with the heterologous fusion proteins (GST-CPAF, GST-HSP60, and GST-IncA) in the western blot assay (Figure 2A). Furthermore, when the anti-CT440 antibody reacted with cells transfected with the recombinant $\mathrm{pDSRedC1/CT440}$ or $\mathrm{pDSRedC} 1$ vectors, strong signals were detected in the cytoplasm of the transfected cells expressing RFP-CT440 but not in the cells expressing RFP, suggesting that the anti-CT440 antibody recognized the RFP-CT440 fusion protein (Figure 2B). The specificity was further verified by a preabsorption experiment which showed that the anti-CT440 protein signals were removed by preabsorption of the antibodies with the GST-CT440 fusion protein, but not the GST-IncA fusion protein (Figure 2C). The results from all the experiments described above convincingly demonstrate that the antibody raised from the CT440 fusion protein was specific to the
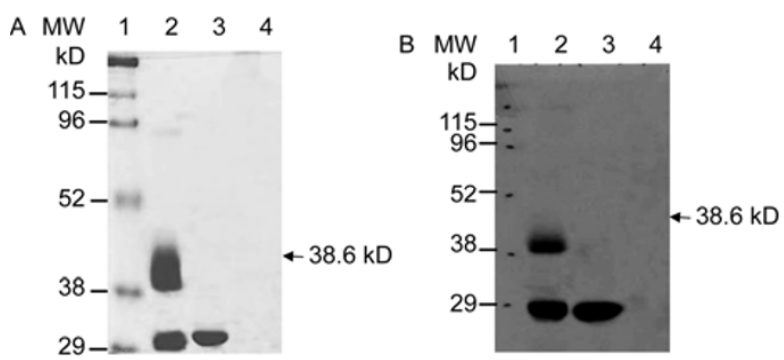

Figure 1 Purification and identification of the CT440 recombinant protein. A, Bacteria harboring either the pGEX-6p/CT440 or empty vector were induced with IPTG. The proteins were purified with glutathione agarose beads and resolved on an SDS gel. The protein bands were visualized using Coomassie brilliant blue staining. B, The SDS gel-resolved protein bands (from panel A) were blotted onto a nitrocellulose membrane for reaction with anti-GST MAb. The specific bands were observed on the membrane, and the molecular weight of the CT440 fusion protein was approximately $38.6 \mathrm{kD}$. Lane 1, Molecular weight markers; lane 2, purified products from XL1-Blue E. coli transformed with the pGEX-6p/CT440 recombinant plasmid; lane 3, purified products from XL1-Blue E. coli transformed with the pGEX-6p vector; lane 4, lysate of XL1-Blue E. coli. 

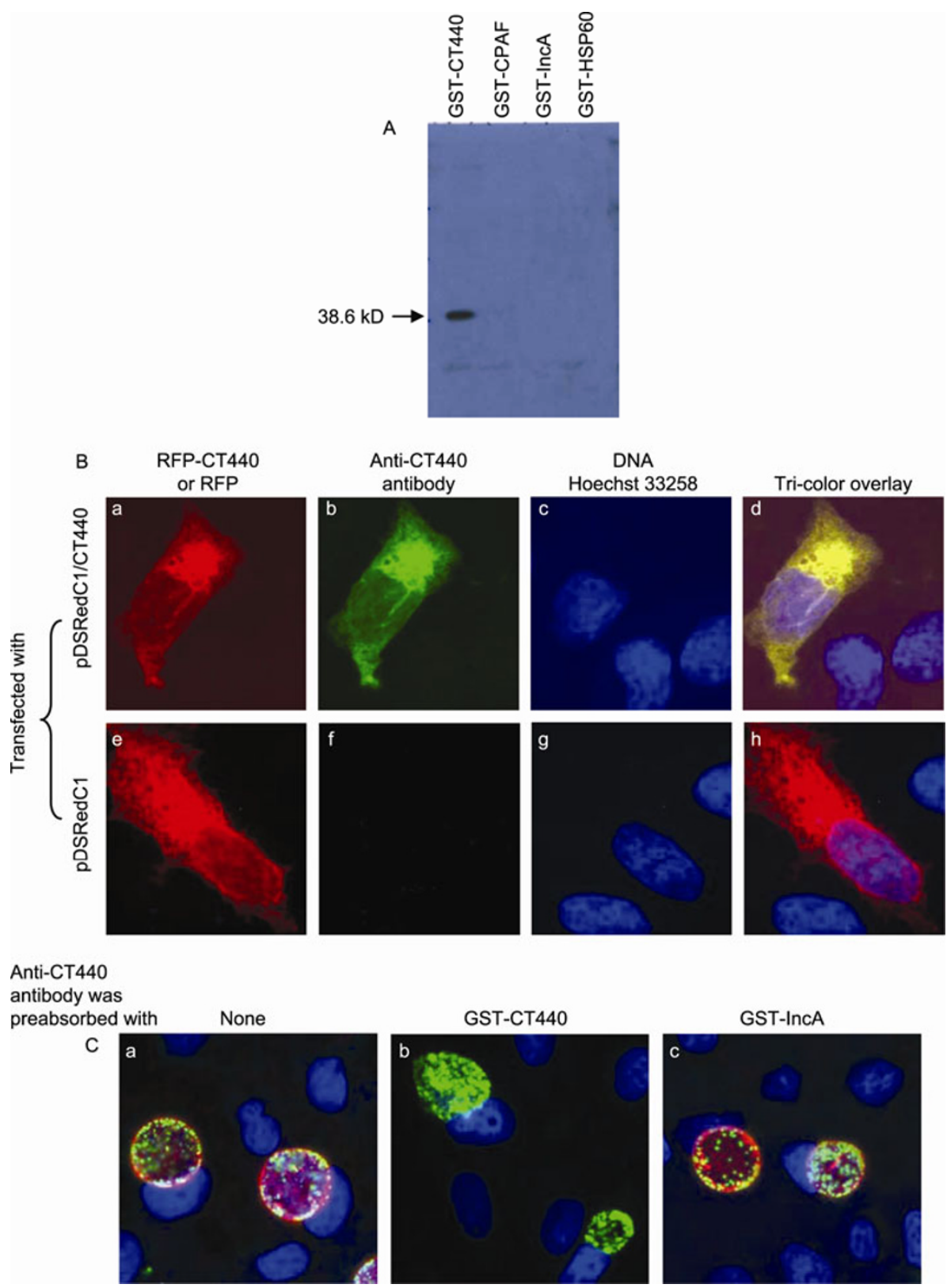

Figure 2 Determination of the specificity of the antibody raised with the GST-CT440 fusion protein. A, Western blot assay. The CT440 fusion protein was resolved in an SDS gel that was also loaded with the same amounts of GST-IncA, GST-CPAF, and GST-HSP60 as the controls. B, Immunofluorescence assay. HeLa cells were transfected with either the pDSRedC1/CT440 or pDSRedC1 vector to express the RFP-CT440 or RFP protein respectively. The expressed proteins were visualized via either the RFP tag (red, images a and e) or the mouse anti-CT440 protein antibody (green, image b). Hoechst 33258 was used to visualize DNA (blue, images $\mathrm{c}$ and g). The antibody raised from the GST-CT440 fusion protein reacted positively with the RFP-CT440 fusion protein (images $b$ and d) but not with the RFP protein (images $f$ and $h$ ). C, Preabsorption experiment. The antibody against the CT440 protein reacted with Chlamydia-infected cells without (image a) or with preabsorption using the GST-CT440 (image b) and GST-IncA (image c) fusion proteins. Whether or not the antibody preabsorbed was visualized with a Cy3-conjugated goat anti-mouse secondary antibody (red). The chlamydial organisms were visualized with a combination of rabbit anti-chlamydial and goat anti-rabbit-Cy2 conjugate antibodies (green). Nuclear DNA was visualized with Hoechst DNA dye (blue). It is clear that the antibody binding to the endogenous CT440 protein was removed by preabsorption with GST-CT440 but not with GST-IncA fusion protein.

\section{CT440 protein.}

\subsection{Localization of CT440 in the inclusion membrane of $C$. trachomatis-infected cells}

The antibody raised with the CT440 fusion protein was used to determine its localization in the Chlamydia-infected cells
(Figure 3). Serovar D-infected HeLa cells were stained with anti-CT440, anti-IncA, anti-CPAF or anti-Hsp60 antibodies. We found that the anti-CT440 antibody detected strong signals in the inclusion membrane under a conventional fluorescence microscope and that the staining pattern was similar to that of the antibody specifically recognizing IncA, a known chlamydial inclusion membrane protein. As the 

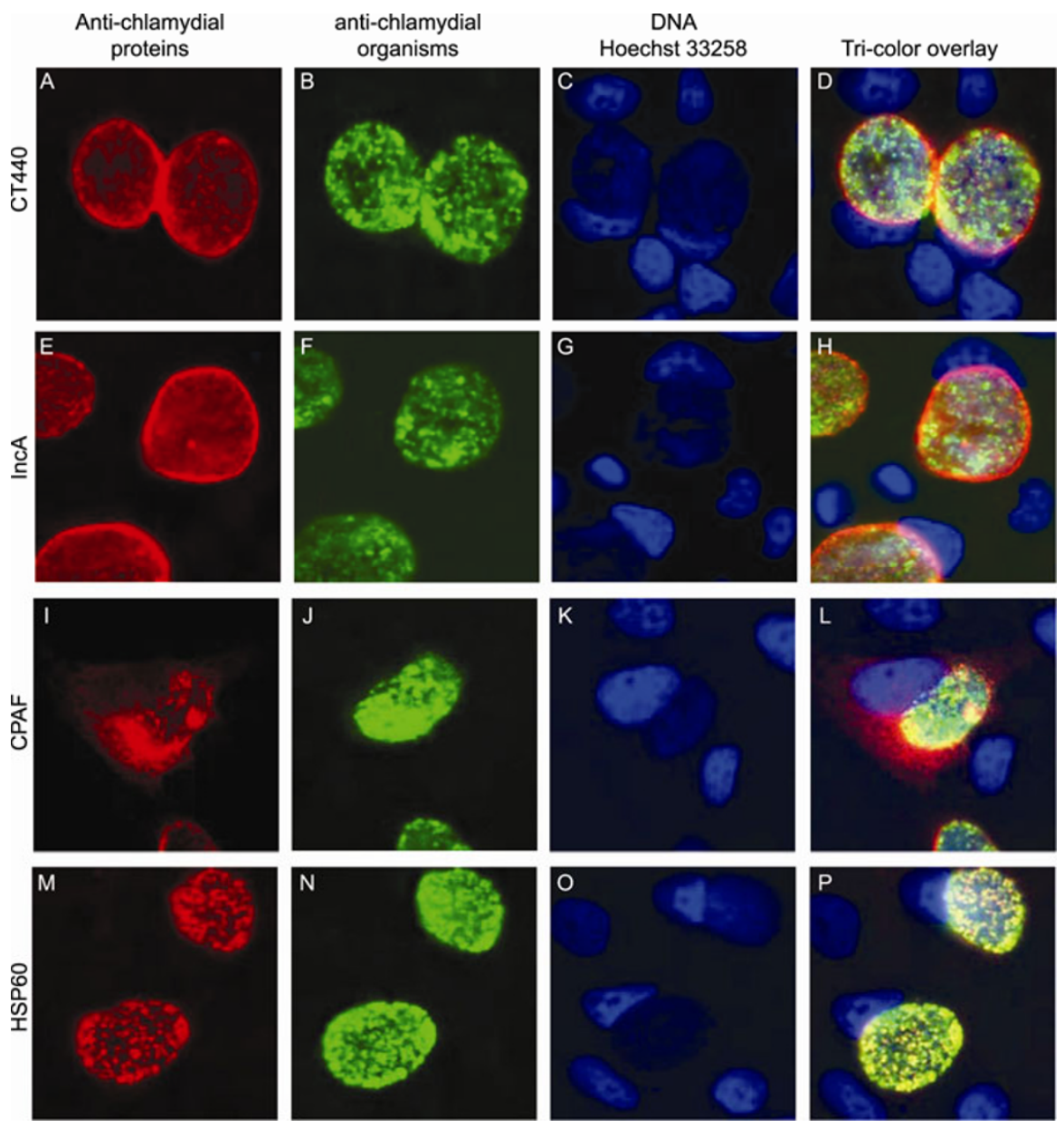

Figure 3 Localization of the CT440 protein in the chlamydial inclusion membrane. HeLa cells infected with C. trachomatis D serovar for $40 \mathrm{~h}$ were processed for immunofluorescence staining. The infected HeLa cells were probed with anti-CT440 (A), anti-IncA (E), anti-CPAF (I) and anti-HSP60 (M) and visualized with a Cy3-conjugated goat anti-mouse immunoglobulin $\mathrm{G}(\mathrm{IgG})$ (red). Rabbit anti-chlamydial antisera together with Cy2-conjugated goat anti-rabbit IgG (green) were used to visualize $C$. trachomatis cells (B, F, J, N). Hoechst 33258 was used to visualize DNA (C, G, K, O). The results showed that anti-CT440 detected strong signals in the inclusion membrane with a pattern similar to that of the anti-IncA (E and H) but not the anti-CPAF (I and L) or anti-HSP60 (M and P) antibodies.

controls, the anti-CPAF antibody detected the cytosol of Chlamydia-infected cells and the anti-HSP60 antibody labeled the intra-inclusion organisms. These results demonstrated that CT440 is an Inc protein.

\subsection{Time course expression of CT440 during chlamy- dial infection}

Using the specific antibody raised with the CT440 fusion protein, we evaluated the expression pattern of the inclusion membrane protein CT440 during chlamydial infection. CT440 expression can be seen early in the developmental cycle (12 $\mathrm{h}$ post-infection) and it was secreted to the inclusion membrane (Figure 4). After it became detectable, CT440 remained in the inclusion membrane throughout the course of the infection, suggesting that CT440 protein may play an essential role in chlamydial interactions with host cells either by supporting the inclusion membrane structure and/or by mediating/regulating the interactions.

\subsection{Expression of CT440 in the inclusion membrane does not affect the subsequent chlamydial infection}

To evaluate the potential effect of CT440 expression on the subsequent chlamydial infection, pDSRedC1/CT440 recombinant plasmid and $\mathrm{pDSRedC} 1$ vector were transfected into HeLa cells. At $12 \mathrm{~h}$ after transfection, the HeLa cells were infected with $C$. trachomatis serovar D and the rates of inclusion-forming units were calculated in transfected and untransfected cells $24 \mathrm{~h}$ after infection. The transfected cells were equally susceptible to the chlamydial infection regardless of whether the cells expressed RFP alone or the RFP-CT440 fusion protein (Figure 5A). The HeLa cells expressing the RFP-CT440 fusion protein displayed an infection rate of about $51 \% \pm 6.1 \%$ while the untransfected HeLa cells on the same coverslip had an infection rate of $56 \% \pm$ $5.9 \%$ in the C. trachomatis-infected culture (Figure 5B). HeLa cells transfected with the RFP vector alone displayed an infection rate of about $64 \% \pm 7.2 \%$ in the RFP-positive 
$0 \mathrm{~h}$

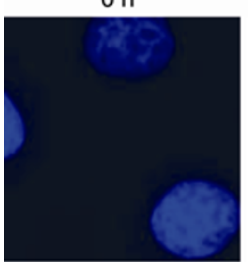

$24 \mathrm{~h}$

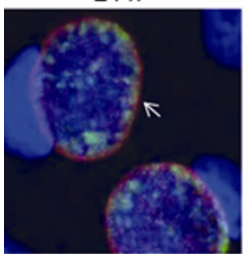

$6 \mathrm{~h}$

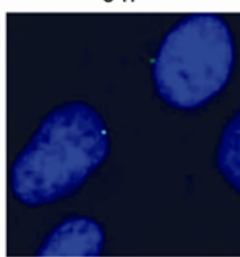

$36 \mathrm{~h}$

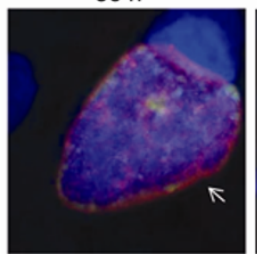

$12 \mathrm{~h}$

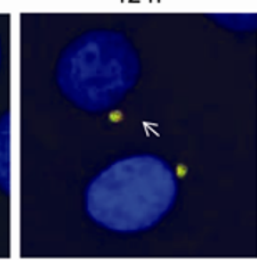

$48 \mathrm{~h}$

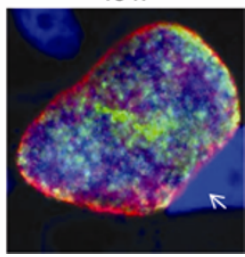

Figure 4 Time course expression of the CT440 protein during chlamydial infection. The HeLa cell samples infected with $C$. trachomatis were processed at various times after infection for immunofluorescence staining with the primary mouse anti-CT440. The mouse antibody stains were visualized with a goat anti-mouse immunoglobulin G (IgG) conjugated with Cy3 (red). The chlamydial organisms were visualized with anti-chlamydial antibody plus a Cy2-conjugated goat anti-mouse IgG (green). DNA was stained with Hoechst 33258 (blue). CT440 expression (indicated by arrows) can be seen early in the developmental cycle ( $12 \mathrm{~h}$ post-infection) and at all other times after that throughout the cycle.
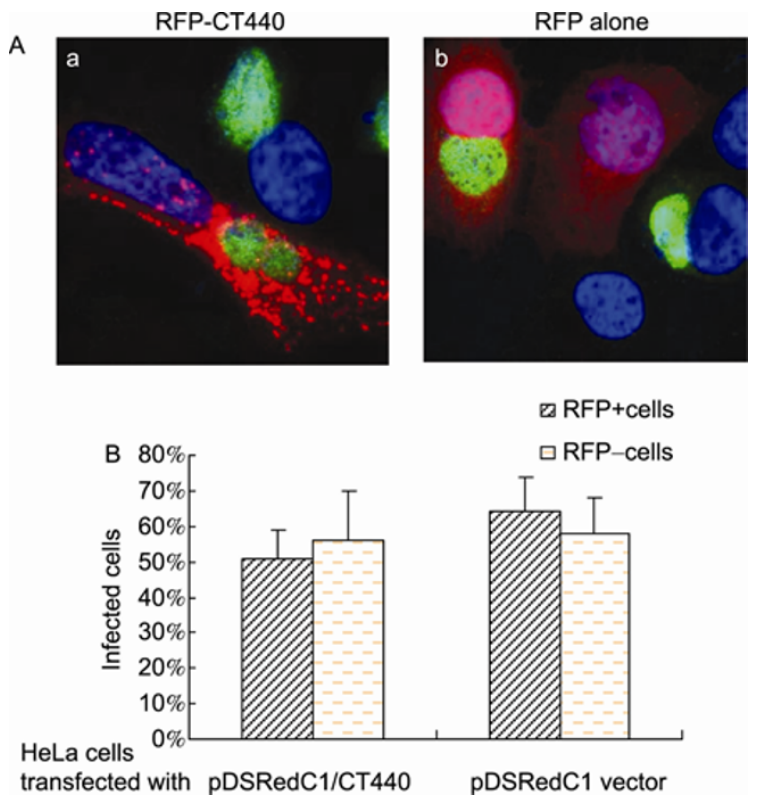

Figure 5 Effect of cytosolic expression of CT440 on the subsequent chlamydial infection. HeLa cells transfected with pDSRedC1/CT440 plasmids or pDSRedC1vector were infected with $C$. trachomatis serovar D organisms. At $24 \mathrm{~h}$ after the infection, the cell samples were processed for immunostaining with a rabbit anti-chlamydial antibody plus a goat anti-rabbit IgG conjugated with $\mathrm{Cy} 2$ (green) and a DNA dye (blue). RFP-CT440 fusion protein was visualized with RFP (red). A, Images of HeLa cells transfected with pDSRedC1/CT440 (image a) and pDSRedC1 (image b). B, Quantitative results, the rates of chlamydial infection in RFP-positive cells and RFP-negative cells from the same cultures were calculated separately. No significant difference between the transfected and untransfected cells $(P>0.05)$ was found. The data is from three independent experiments.

cells and $58 \% \pm 6.3 \%$ in the RFP-negative cells. No significant difference in the chlamydial infection rates of these two cell populations in either HeLa cells transfected with the pDSRedC1 vector or those transfected with the pDSRedC1CT440 plasmid $(P>0.05)$ was found, demonstrating that prior expression of RFP or RFP-CT440 fusion proteins in HeLa cells did not alter the susceptibility of the transfected cells to the subsequent chlamydial infection. The above observations suggested that cytosolic CT440 may not directly alter chlamydial intracellular replication but it may participate in chlamydial interactions with host cells.

\section{Discussion}

Inc proteins play potentially important roles in chlamydial biology and pathogenesis and various approaches have been developed to identify new Inc proteins. Based on the hydropathy profiles of the known Inc proteins, about $50 \mathrm{C}$. trachomatis hypothetical proteins have been predicted to be Inc proteins. However, not all predicted proteins are localized in the inclusion membranes and not all known Inc proteins can be predicted [21-24]. Therefore, additional experimental evidence needs to be found to validate the prediction, and greater efforts need to be made to search for new Inc proteins in Chlamydia-infected cells.

Using an anti-fusion protein antibody approach to localize endogenous chlamydial proteins, we found that the hypothetical protein CT440 was localized in the C. trachomatis inclusion membrane. To the best of our knowledge, this is the first time that this has been reported. Although antibodies raised with fusion proteins do not always efficiently recognize the corresponding endogenous proteins, in the present study, we have presented compelling evidence to show that the anti-CT440 staining of the inclusion membrane is specific to the CT440 protein. First, while the anti-CT440 antibody reacted positively with the GST-CT440 fusion protein, it did not react with the GST-IncA, GST-CPAF, or GST-HSP60 fusion proteins in a Western blot assay. Despite the fact that all fusion proteins contained a common GST fusion tag, the anti-CT440 fusion protein antibody failed to recognize GST in unrelated fusion proteins. Second, the anti-CT440 antibody reacted with the RFP-CT440 fusion protein expressed in transfected cells, but not with RFP alone. Finally, the inclusion membrane staining by the anti-CT440 antibody was blocked by preabsorption of the antibody with the GST-CT440 fusion protein, but not with any of the other heterologous fusion proteins. Together, the above experiments demonstrated that the anti-CT440 antibody specifically detected the CT440 protein in the inclusion membrane of the C. trachomatis infected cells.

From their localization at the interface between the bacteria and its host cell, Inc proteins may play potential roles in chlamydial pathogenesis. However, only a few functions have been described for these proteins and, for the most part, their exact functions in infection are unknown. The identi- 
fication of CT440 as an Inc protein leads to the next step which is to determine its potential biological functions. Because of the lack of genetic tools for manipulating chlamydial genomes, approaches for analyzing chlamydial protein functions are limited. In the present study, using a specific anti-CT440 antibody, we studied the effect of CT440 expressed via a transgene on subsequent chlamydial infection. It was shown previously that prior expression of IncA in host cell cytosol inhibited the subsequent chlamydial infection [25,26]; however, in our study we found that HeLa cells were equally susceptible to chlamydial infection regardless of whether or not pre-existing cytosolic CT440 fusion protein was present. This result indicated that CT440 may play a role different from that of IncA in C. trachomatis biology. We also found that CT440 became detectable $12 \mathrm{~h}$ after infection and once it became detectable it remained in the inclusion membranes throughout the course of the infection, suggesting that CT440 may play essential roles in chlamydial interactions with host cells either by supporting the inclusion membrane structure or/and by mediating the interactions between Chlamydia and the host cells. We are currently investigating further the structural feature of the CT440 inclusion membrane protein and its roles in $C$. trachomatis pathogenesis.

In conclusion, the present study demonstrated that the hypothetical protein CT440 was localized in the inclusion membrane of Chlamydia-infected cells and became detectable $12 \mathrm{~h}$ after chlamydial infection and remained in the inclusion membranes throughout the course of the infection. Our data revealed that the pre-existence of cytosolic CT440 fusion protein in the cells did not affect the subsequent Chlamydia infection, suggesting that the CT440 protein may participate in the chlamydial interactions with host cells.

This work was supported by the National Natural Science Foundation of China (Grant Nos. 30970165 and 81102230), the Hunan Provincial Natural Science Foundation of China (Grant No. 09JJ3059), and the Team Project for the Technology Innovation of Higher Education of Hunan Province, China.

1 Mabey D C, Solomon A W, Foster A. Trachoma. Lancet, 2003, 362: 223-229

2 Brunham R C, Rey-Ladino J. Immunology of Chlamydia infection: implications for a Chlamydia trachomatis vaccine. Nat Rev Immunol, 2005, 5: 149-161

3 Belland R, Ojcius D M, Byrne G I. Chlamydia. Nat Rev Microbiol, 2004, 2: 530-531

4 Kilmarx P H, Mock P A, Levine W C. Effect of Chlamydia trachomatis coinfection on HIV shedding in genital tract secretions. Sex Transm Dis, 2001, 28: 347-348

5 Mbizvo E M, Msuya S E, Stray-Pedersen B, et al. HIV seroprevalence and its associations with the other reproductive tract infections in asymptomatic women in Harare, Zimbabwe. Int J STD AIDS, 2001, 12: 524-531

6 Smith J S, Muñoz N, Herrero R, et al. Evidence for Chlamydia trachomatis as a human papillomavirus cofactor in the etiology of invasive cervical cancer in Brazil and the Philippines. J Infect Dis, 2002, 185: 324-331

7 Wallin K L, Wiklund F, Luostarinen T, et al. A population-based prospective study of Chlamydia trachomatis infection and cervical carcinoma. Int J Cancer, 2002, 101: 371-374

8 Hackstadt T. The diverse habitats of obligate intracellular parasites. Curr Opin Microbiol, 1998, 1: 82-87

9 Hackstadt T, Fischer E R, Scidmore M A, et al. Origins and functions of the chlamydial inclusion. Trends Microbiol, 1997, 5: 288-293

10 Abdelrahman Y M, Belland R J. The chlamydial developmental cycle. FEMS Microbiol Rev, 2005, 29: 949-959

11 Carabeo R A, Mead D J, Hackstadt T. Golgi-dependent transport of cholesterol to the Chlamydia trachomatis inclusion. Proc Natl Acad Sci USA, 2003, 100: 6771-6776

12 Su H, McClarty G, Dong F, et al. Activation of Raf/MEK/ERK/cPLA2 signaling pathway is essential for chlamydial acquisition of host glycerophospholipids. J Biol Chem, 2004, 279: 9409-9416

13 Zhong G. Chlamydia trachomatis secretion of proteases for manipulating host signaling pathways. Front Microbiol, 2011, 2: 14

14 Li Z, Chen D, Zhong Y, et al. The chlamydial plasmid-encoded protein pgp3 is secreted into the cytosol of Chlamydia-infected cells. Infect Immun, 2008, 76: 3415-3428

15 Lei L, Qi M, Budrys N, et al. Localization of Chlamydia trachomatis hypothetical protein CT311 in host cell cytoplasm. Microb Pathog, 2011, 51: 101-109

16 Qi M, Gong S, Lei L, et al. A Chlamydia trachomatis OmcB C-terminal fragment is released into the host cell cytoplasm and is immunogenic in humans. Infect Immun, 2011, 79: 2193-2203

17 Li Z, Wu Y, Huang Q, et al. Localization and characterization of hypothetical protein CT358 in the Chlamydia trachomatis-infected cells. Prog Biochem Biophys, 2009, 36: 549-554

18 Li Z, Zhong Y, Lei L, et al. Antibodies from women urogenitally infected with $C$. trachomatis predominantly recognized the plasmid protein pgp3 in a conformation-dependent manner. BMC Microbiol, 2008, 8: 90

19 Xiao Y, Zhong Y, Su H, et al. NF-kappa B activation is not required for Chlamydia trachomatis inhibition of host epithelial cell apoptosis. J Immunol, 2005, 174: 1701-1708

20 Li Z, Chen C, Chen D, et al. Characterization of fifty putative inclusion membrane proteins encoded in the Chlamydia trachomatis genome. Infect Immun, 2008, 76: 2746-2757

21 Bannantine J P, Griffiths R S, Viratyosin W, et al. A secondary structure motif predictive of protein localization to the chlamydial inclusion membrane. Cell Microbiol, 2000, 2: 35-47

22 Rockey D D, Scidmore M A, Bannantine J P, et al. Proteins in the chlamydial inclusion membrane. Microbes Infect, 2002, 4: 333-340

23 Fling S P, Sutherland R A, Steele L N, et al. $\mathrm{CD}^{+} \mathrm{T}$ cells recognize an inclusion membrane-associated protein from the vacuolar pathogen Chlamydia trachomatis. Proc Natl Acad Sci USA, 2001, 98: 1160-1165

24 Toh H, Miura K, Shirai M, et al. In silico inference of inclusion membrane protein family in obligate intracellular parasites chlamydiae. DNA Res, 2003, 10: 9-17

25 Alzhanov D, Barnes J, Hruby D E, et al. Chlamydial development is blocked in host cells transfected with Chlamydophila caviae incA. BMC Microbiol, 2004, 4: 24

26 Delevoye C, Nilges M, Dautry-Varsat A, et al. Conservation of the biochemical properties of IncA from Chlamydia trachomatis and Chlamydia caviae: oligomerization of IncA mediates interaction between facing membranes. J Biol Chem, 2004, 279: 46896-46906

Open Access This article is distributed under the terms of the Creative Commons Attribution License which permits any use, distribution, and reproduction in any medium, provided the original author(s) and source are credited. 